\title{
Abordagem situacional do enfermeiro no exame fisico hematológico: uma reflexao com faye abdellah
}

\section{Situational approach of the nurse in hematological physical exami- nation: a reflection with faye abdellah}

\author{
Vinícius Rodrigues de Souza' • Gisella de Carvalho Queluci $\bullet$ Amanda Ribeiro Mendonça ${ }^{3}$ \\ Suelem Couto Frian Dias ${ }^{4} \bullet J^{\text {Juliane da Silveira Jasmim }}{ }^{5}$
}

\begin{abstract}
RESUMO
Objetivou-se refletir acerca de como o enfermeiro deverá realizar o exame físico em clientes hematológicos a partir da compreensão de uma situação problema. Trata- se de um estudo de reflexão fundamentado na base teórico-filosófica de Faye Abdellah, discutindo pontos principais desta linha de pensamento diante de uma situação problema. Os enfermeiros devem usar o método de solução dos problemas-chave de enfermagem relacionada às necessidades de saúde das pessoas. Os problemas podem ser evidentes, através de uma condição aparente, ou ser encobertos, quando relacionados às esferas emocionais, sociológicas e interpessoais por natureza. Conclui-se que se faz necessário que os enfermeiros, na realização do exame físico, identifiquem e resolvam os problemas, satisfazendo as necessidades do cliente. Palavras-chave: Enfermagem; Exame físico; Hematologia.
\end{abstract}

\begin{abstract}
The objective was to reflect on how the nurse should perform the physical examination in hematological clients from the understanding of a problem situation. It is a study of reflection based on the theoretical-philosophical basis of Faye Abdellah, discussing main points of this line of thought in the face of a problem situation. Nurses should use the method of solving key nursing problems related to people's health needs. Problems may be evident, through an apparent condition, or be overt, when related to the emotional, sociological, and interpersonal spheres by nature. It is concluded that it is necessary for the nurses, in performing the physical examination, to identify and solve the problems, satisfying the needs of the client.
\end{abstract}

Keywords: Nursing; Physical examination; Hematology.

'Mestre em Ensino na Saúde pela Universidade Federal Fluminense; Enfermeiro; viniciussouza.enf@gmail.com.

2Professora Adjunta da Universidade Federal Fluminense; Enfermeira; gisellaqueluci@yahoo.com.br

${ }_{3}^{3}$ Mestre em Ensino na Saúde pela Universidade Federal Fluminense; Enfermeira; amanda-mendonca@hotmail.com

${ }^{4}$ Mestre em Ensino na Saúde pela Universidade Federal Fluminense; Fonoaudióloga; suelemfrian@gmail.com

${ }^{5}$ Mestre em Ensino na Saúde pela Universidade Federal Fluminense; Enfermeira; Juliane_jasmim@hotmail.com 


\section{INTRODUÇÃO}

$O$ processo de enfermagem representa o principal modelo metodológico para o desempenho sistemático da prática profissional, ou instrumento tecnológico de que se lança mão para favorecer o cuidado, para organizar as condições necessárias à realização do cuidado e para documentar a prática profissional. Esse processo é organizado em etapas interdependentes, sendo elas: histórico de enfermagem (composto por anamnese e exame físico), diagnósticos de enfermagem, prescrição e implementação dos cuidados, avaliação e registro das atividades realizadas ${ }^{(1)}$.

Sendo assim, o exame físico precedido pela anamnese compõe a fase do histórico de enfermagem. Refere-se a uma revisão céfalo-caudal do corpo humano para a investigação de cada sistema corporal do cliente, por meio da inspeção, da palpação, da percussão, da ausculta, do olfato e do uso de alguns instrumentos e aparelhos, fornecendo informações objetivas sobre o corpo e permitindo que o enfermeiro faça julgamentos clínicos ${ }^{(2)}$.

No que tange a avaliação de clientes com alterações hematológicas, esses apresentam agravos ao sistema imunológico que advêm tanto da própria patologia quanto do regime terapêutico. Logo, a atuação do enfermeiro deve visar à prevenção e a detecção precoce das complicações, pontos fundamentais da assistência com vistas a garantir a sobrevida do indivíduo.

Sabe-se que, na prática diária do enfermeiro, seu cliente necessita de cuidados fundamentados em conhecimentos específicos que determinam as intervenções de enfermagem visando à resolução dos problemas encontrados, em uma perspectiva profissional. Nas instituições de saúde, os enfermeiros, ao prestarem assistência, deparam-se com uma realidade na qual a população requer medidas de cuidado, que envolvem, antes de tudo, a identificação e resolução dos problemas de saúde dos clientes. Assim, a aprendizagem relacionada ao cuidado baseado nas necessidades do cliente se faz necessária. Isto porque os enfermeiros, estando comprometidos com o desenvolvimento de um cuidado peculiar e direcionado, e simultaneamente trabalhando com competência, são capazes de mudar as alterações hematológicas encontradas. Ou seja, por meio do conhecimento, acabam por reformular sua prática profissional, contribuindo, então, para mudanças qualitativas nas situações do cliente.

Logo, pode-se considerar que confere à Enfermagem o sentido de problema, aqueles elementos que precisam ser solucionados pelo enfermeiro. Nestes determinantes, a expressão "situação-problema" envolve muitos elementos, abrangem aspectos que transcendem o que se pode perceber, o que conhecemos ou vivenciamos em qualquer momento da vida ${ }^{(3)}$.

Situações de Enfermagem são o conjunto de circuns- tâncias da realidade espacial e temporal que afetam a saúde de indivíduos, grupos ou comunidades e em cuja esfera ocorre o encontro do enfermeiro com seu clien$\mathrm{te}^{(4)}$. Com isso, nota-se que as situações problema diferem, às vezes, quanto ao grau de complexidade não apenas porque são relacionadas com a sintomatologia do cliente, mas talvez pela soma das dificuldades na proporção de elementos presentes na trama do que ocorre em torno do cliente, elementos percebidos na experiência prática e que dão o sentido de problema à situação de enfermagem - entendida também como situação clínico -assistencial - que envolve o cliente, com certo grau de complexidade $^{(3)}$.

Sendo assim, a aplicação das teorias de enfermagem no âmbito da assistência promove a construção de um conhecimento mais sólido, crítico e reflexivo, proporcionando cientificidade à profissão, aprimorando as habilidades teórico-práticas e contribuindo com melhoria do cuidado prestado.

Somado a isso, a teoria dos 21 problemas de Enfermagem de Faye Abdellah descreve requisitos da função do enfermeiro e os objetivos terapêuticos da profissão, em seu desenvolvimento, e tiveram como foco as principais necessidades do cliente e os problemas de enfermagem.

Os enfermeiros devem usar o método de solução dos problemas-chave de enfermagem relacionados às necessidades de saúde das pessoas ${ }^{(3)}$. Os problemas podem ser evidentes, através de uma condição aparente, ou ser encobertos, quando relacionados às esferas emocional, sociológica e interpessoal por natureza.Abdellah enfatiza que a assistência de enfermagem deve considerar o indivíduo como um todo, inclusive suas necessidades físicas, sociais, psicológicas e espirituais e as da sua família ${ }^{(5)}$.

Os 21 problemas descritos por Abdellah são: - Manter boa higiene e conforto físico; -Promover a atividade ideal: exercício, repouso e sono; - Promover a segurança através da prevenção de acidentes, ferimentos ou outros traumatismos e através da prevenção da disseminação de infecção; - Manter a mecânica do corpo e prevenir e corrigir as deformidades; - Facilitar a manutenção de um suprimento de oxigênio para todas as células do corpo; - Facilitar a manutenção da nutrição de todas as células do corpo; - Facilitar a manutenção da eliminação; - Facilitar a manutenção do equilíbrio hídrico e eletrolítico; - Reconhecer as reações fisiológicas do corpo às condições da doença - patológicas, fisiológicas e compensatórias; - Facilitar a manutenção dos mecanismos das funções reguladoras; - Facilitar a manutenção da função sensorial; - Identificar e aceitar as expressões positivas e negativas, os sentimentos e as reações; - Identificar e aceitar o inter-relacionamento de emoções em doenças orgânicas; - Facilitar a manutenção efetiva da comunicação verbal e não-verbal; - Promover o desenvolvimento 
de relacionamentos interpessoais produtivos; - Facilitar o progresso em direção à obtenção de metas espirituais pessoais; - Criar e/ou manter um ambiente terapêutico; - Facilitar a conscientização de si mesmo como indivíduo com necessidades físicas, emocionais e de desenvolvimento variadas; - Aceitar as metas ideais possíveis à luz das limitações físicas e emocionais; - Usar os recursos comunitários como auxílio na resolução dos problemas que surgem com a doença e - Compreender o papel dos problemas sociais como influentes no caso de doença $a^{(5)}$.

Assim, a teórica classifica os principais problemas de enfermagem, alguns como evidentes e outros não evidentes, e afirma que o desenvolvimento das ações de enfermagem consiste na resolução desses problemas ${ }^{(5)}$.

Segundo Abdellah ${ }^{(5)}$, os problemas de enfermagem evidentes condizem com condições enfrentadas pelo cliente e família, e nas quais o enfermeiro pode assisti -los, ou ajudá-los, mediante o desempenho de suas funções profissionais (por exemplo: um cliente constipado, apresentando abdômen distendido com dor a palpação requer cuidado pela enfermeiro para promover uma meIhora na motilidade gastrointestinal). Mas não é só isto. Um problema de enfermagem não evidente confere com uma condição oculta, escondida, encoberta, mas enfrentada pelo cliente, às vezes sentida pela família, e em face da qual o enfermeiro pode assisti-los ao desempenhar suas funções profissionais (por exemplo: um indivíduo com comunicação verbal prejudicada relacionado a distúrbios emocionais) ${ }^{(1)}$.

Logo, encontra-se a relevância do estudo na medida em que o mesmo reforça a importância da abordagem situacional em clientes hematológicos hospitalizados. A assistência de enfermagem centrada no cliente facilita a compreensão das situações-problema, trazendo um enfoque mais abrangente em relação ao que ocorre quando são exclusivamente voltadas para as patologias e as causas da hospitalização.

A reflexão foi conduzida a partir dos questionamentos: Como realizar o exame físico nos clientes hematológicos hospitalizados a partir de uma abordagem situacional? Quais as dimensões do cliente hematológico devem ser exploradas? Como resolver os problemas de enfermagem diante da teoria de Faye Abdellah?

Diante do exposto, este artigo tem como objetivo refletir acerca de como o enfermeiro deverá realizar o exame físico em clientes hematológicos a partir da compreensão de uma situação problema a luz da teoria de Faye Abdellah.

\section{MÉTODOS}

Trata-se de um estudo de reflexão fundamentado na base teórica-filosófica de Faye Abdellah, que retrata a identificação dos problemas de enfermagem, totalizando em vinte e um e que estão relacionados ao biológico, psicológico e social do indivíduo, identificados através da observação direta das necessidades evidentes e da comunicação e interação com o cliente para as necessidades não evidentes. A escolha destes pontos deu-se por sua ligação direta com a discussão proposta. A identificação dos problemas propostos pela teórica é extremamente necessário para a tomada de decisão do enfermeiro, considerando-se, assim, oportuno o destaque de algumas de suas resoluções.

A partir de discussões, autores e materiais trabalhados nas aulas do Mestrado Profissional de Ensino na Saúde, da Universidade Federal Fluminense (UFF), durante os anos de 2017 e 2018 , surgiu a ideia de desenvolver a respectiva reflexão.

\section{RESULTADOS E DISCUSSÃO}

Buscou-se expor a importância de uma abordagem situacional e identificação dos problemas de enfermagem no exame físico do cliente hematológico. A partir dos questionamentos balizadores desta reflexão, o texto foi organizado diante da temática "A evidência dos problemas de enfermagem no exame físico". Os argumentos esclarecem que a partir de uma abordagem situacional, é possível a identificação dos 21 problemas propostos pela teórica do estudo, bem como a resolução dos mesmos.

\section{A evidência dos problemas de enfermagem no exame físico}

A realização do exame físico em clientes hematológicos a partir de uma abordagem situacional envolve a observação de elementos que influenciam a realização do procedimento. $O$ enfermeiro deve ser capaz de atuar nas diferentes realidades espaciais e temporais, saber priorizar e tomar providências nas tomadas de decisão, com o fim na melhoria da assistência.

Logo, o exame físico, assim como qualquer outro procedimento realizado pelo enfermeiro, sofre influencias diversas dentro de um cenário de saúde, não se limitando somente ao conhecimento teórico prático para a execução. Tais influências de cunho emocional, psicológico, gerencial, ambiental, entre outras, precisam ser resolvidas para que não prejudique o cuidado realizado(6). Diante disso, realizar uma abordagem situacional compreende aprender e compreender todas as barreiras e impedimentos que podem surgir ao longo de uma assistência, tendo a iniciativa e conhecimento na priorização das situações.

A exemplo de outros clientes, o portador de alterações hematológicas requer uma assistência de enfermagem especializada na qual o enfermeiro o veja nas dimensões bio-psico-social. Desse modo, ele se transforma no centro de suas intervenções, com vistas à sua adaptação 
e ao autocuidado. Por isso, um registro fiel das informações sobre a evolução clínica faz diferença no cuidado do cliente hematológico e a monitorização dos sinais e sintomas que a doença provoca. Com isso, os enfermeiros, como membros da equipe de saúde, assumem função vital na recuperação do cliente ${ }^{(7)}$.

Diante da imagem abaixo, é possível refletir que durante a realização do exame físico, problemas de enfermagem podem ser identificados. Tais problemas podem ainda ser classificados como evidentes ou não evidentes a depender da condição do cliente estar aparente ou oculta.

As doenças hematológicas se expressam por alterações localizadas no sangue ou em tecidos formadores dele. Fisiologicamente, sabe-se que as alterações que a doença produz e o intenso protocolo de tratamento, traduzem em manifestações de sinais e sintomas debilitadoras ao cliente ${ }^{(8)}$.

Além disso, os clientes hematológicos podem apresentar sinais e sintomas variados, decorrentes de alterações em diversos sistemas orgânicos, pois as modificações presentes na crase sanguínea geram distúrbios na nutrição, oxigenação, coagulação e sistema de defesa do organismo, provocando muitas vezes fraqueza, debilidade física, emagrecimento, febre, lesões ulcerativas de pele e mucosas, parestesias, dores, sangramentos e infecções ${ }^{(9)}$.
Desse modo, avaliando a condição do processo patológico do cliente hematológico, foi possível associar oito problemas de enfermagem segundo Abdellah. Tais problemas permeiam a melhoria do estado fisiológico do cliente em questão como manter a mecânica do corpo e prevenir e corrigir as deformidades; facilitar a manutenção: dos mecanismos das funções reguladoras, da função sensorial, de um suprimento de oxigênio para todas as células do corpo, da nutrição de todas as células do corpo, da eliminação, do equilíbrio hídrico e eletrolítico; além de reconhecer as reações fisiológicas do corpo às condições da doença - patológicas, fisiológicas e compensatórias.

Dissertando no mérito dos problemas de enfermagem não evidentes, estes se apresentam em grande número nos clientes hematológicos. Devido à complexidade e gravidade do quadro de saúde, bem como todo o tratamento agressivo, os clientes de patologias hematológicas acumulam fatores de natureza emocional, sociológica e interpessoal, que são frequentemente percebidos de maneira incorreta ou não percebidos ${ }^{(10)}$.

Por outro lado, o enfermeiro para realizar um exame físico minucioso, precisa quebrar as possíveis barreiras existentes entre a comunicação e o toque, alcançando a confiança e o respeito, executando assim o procedimento que envolve não somente conhecimentos de ana-

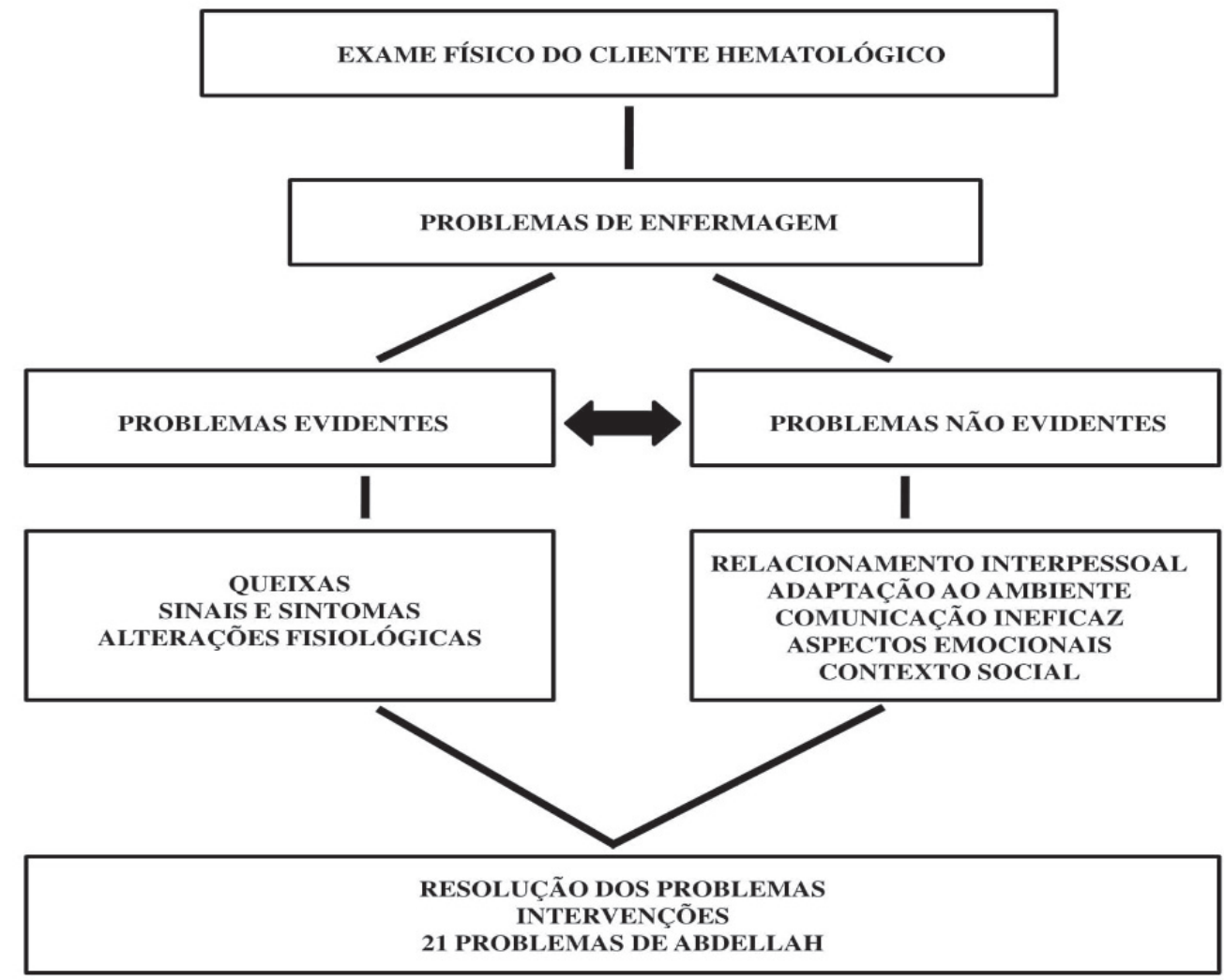

FIGURA 1 - Abordagem Situacional do Enfermeiro no Exame Físico do Cliente Hematológico. Rio de Janeiro, RJ, Brasil, 2018. 
tomia e fisiologia, mas também percepções humanísticas que envolve o respeito, a atenção, a escuta qualificada, entre outros aspectos. É necessário que se promova um melhor relacionamento interpessoal com os clientes, gerando um sentimento de confiança entre o assistido e aquele que assiste ${ }^{(I I)}$.

Ademais, um cliente que vivencia uma internação hospitalar num cenário hematológico pode presenciar diversas situações que influenciam negativamente na sua saúde emocional como: baixa autoestima, medo da morte, complicações referentes ao diagnóstico e tratamento. Sendo assim, todos os mecanismos que geram tensão e ansiedade devem ser amenizados.

O profissional de Enfermagem pode fazer muito no ambiente hospitalar, com a efetivação de pequenos ajustes e melhores adaptações, para diminuir o estresse dos clientes e satisfazer às suas necessidades ${ }^{(8)}$. A teoria de Florence Nightingale, por exemplo, significa zelar por um ambiente mais calmo e agradável, afastando-se da imagem negativa e carregada de um cenário hospitalar, onde o sofrimento é instaurado(12).

Portanto, ao realizar o exame físico, medidas devem ser implementadas para que nenhum fator externo influencie nos achados do procedimento. A execução do procedimento num local que garanta privacidade e tranquilidade favorece a receptividade do cliente em relação às técnicas realizadas pelo enfermeiro.

Outro ponto importante a ser refletido se trata da comunicação. $O$ cliente portador de alterações hematológicas vivencia ao longo do curso da doença medos e anseios relacionados ao medo da morte, sofrimentos atrelados a complicações e efeitos colaterais do tratamento. Com isso, alguns acabam adquirindo distanciamento das relações sociais, permanecendo isolados, pouco comunicativos e responsivos ${ }^{(13)}$. Tal problemática pode ser sanado no exame físico prestado pelo profissional de saúde. $O$ enfermeiro deve ter a capacidade de melhorar a comunicação do indivíduo, alcançando nos clientes uma sensação de segurança, liberdade e afinidade para assim exporem todas as queixas e angustias presentes.

Reforçando a temática dos problemas não evidentes, a complexidade do tratamento e das diferentes demandas físicas e psíquicas que afligem os clientes hematológicos, além do medo da morte, faz se necessária uma maior atenção às dimensões relacionadas à qualidade de vida desses indivíduos. Com isso, o enfermeiro ao realizar o exame físico precisa estar atento a sinais que indiquem um sofrimento emocional, implementando cuidados como comunicação terapêutica, encorajando os clientes ao longo das etapas do tratamento.

Pensando em questões sociais, os familiares de clientes com acometimentos hematológicos e crônicos tendem a se reorganizar e adaptar-se, pois o enfermo necessitará de cuidados, os papéis e as funções são repensados e redistribuídos para auxiliá-lo em seus sentimentos confusos e dolorosos causados pelo processo de adoecimento. Não só o cliente é abalado pela enfermidade, mas toda a sua rede social principalmente o ciclo familiar devido aos esforços para adaptar-se a situação de doença ${ }^{(14)}$.

Do mesmo modo, o enfermeiro ao realizar o exame físico em clientes hematológicos, deve fornecer instrução ao cliente que objetive ajudá-lo a administrar sua enfermidade crônica, principalmente com a ajuda da família. $O$ cuidado é perpassado, essencialmente, pela relação com o outro, o que exige do familiar que cuida capacidade de enfrentamento e de desprendimento, o que por sua vez, envolve diferentes sentimentos ${ }^{(15)}$.

Sendo assim, aos profissionais de saúde, é fundamental conseguir identificar e avaliar as necessidades específicas do cliente, considerando fatores culturais, religiosos, sociais e psicológicos, além da capacidade da família para assumir o papel de prestadora de cuidados.

Assim também, o cuidado nessas condições deverá considerar o processo de enfrentamento e adaptação pessoal, familiar e comunitária, as experiências prévias; existência de situações similares, tradição cultural e princípios religiosos, na definição diagnóstica e intervenção, bem como a realização do exame físico.

Diante disso, ao contrário das condições agudas nas quais, em geral, pode-se esperar uma recuperação adequada, os clientes hematológicos em condições crônicas levam a mais sintomas e à perda de capacidade funcional que afetam diretamente a qualidade de vida, na qual consiste em um processo de percepção e de satisfação do indivíduo com sua realidade familiar, social e ambiental (16). Sendo assim, esses problemas não evidentes devem ser identificados durante o exame físico e resolvidos durante a implementação dos cuidados.

Com isso, trabalhar com pessoas com doença crônica ou incapacidade exige não somente lidar com os aspectos médicos de seu distúrbio e realização do exame físico, mas também trabalhar com a integralidade, do ponto de vista físico, emocional e social. Essa abordagem holística para o cuidado requer que os enfermeiros empreguem rodo o seu repertório de conhecimentos e competências, inclusive o conhecimento das ciências sociais, em particular a psicologia, muito usada em clientes hematológicos que lidam com dor e sofrimento ${ }^{(17)}$.

Faye Abdellah em sua teoria descreve um método de solução de problemas que permite estruturar um corpo único de conhecimentos e habilidades da Enfermagem relacionada à sua prática profissional ${ }^{(5)}$. Em se tratando dos problemas não evidentes descritos acima, podemos elencar com soluções: criar e/ou manter um ambiente terapêutico; facilitar a manutenção efetiva da comunica- 
ção verbal e não-verbal" para se alcançar resolutividade na assistência prestada aos clientes; facilitar a conscientização de si mesmo como indivíduo com necessidades físicas, emocionais e de desenvolvimento variadas" e "aceitar as metas ideais possíveis à luz das limitações físicas e emocionais"; usar os recursos comunitários como auxílio na resolução dos problemas que surgem com a doença e compreender o papel dos problemas sociais como influentes no caso de doença.

Do mesmo modo, os problemas profissionais da enfermagem são fenômenos que se apresentam na pratica da profissão, são as necessidades que os serem humanos têm e que requerem atuação do profissional enfermeiro para satisfazê-las.

\section{CONCLUSÃO}

Com base nessa reflexão proposta, é possível perceber que a teoria de Abdellah descreve os 21 problemas de enfermagem que estão relacionados ao biológico, psicológico e social do indivíduo e são identificados através da observação direta das necessidades evidentes, da comunicação, e interação com os clientes para as necessidades não evidentes.

A resolução desses problemas expressa à capacida- de de autocuidado e manutenção destes para obter o bem estar. Para isso, se faz necessário que os enfermeiros, durante a realização do exame físico, identifiquem e resolvam os problemas, contemplando assim um estado no qual os clientes tem suas necessidades satisfeitas, sem problemas reais ou potenciais.

Como contribuição dessa reflexão para a Enfermagem, é de extrema importância que os enfermeiros busquem um novo paradigma na atenção à saúde dos clientes hematológicos, centrado na visão holística do usuário como sujeito protagonista de direitos, bem como na atenção articulada e humanizada e atitude ética nas relações intersubjetivas, além de utilizar estratégias de resolução de situações problemas que favorecem o pensamento crítico .

Um pequeno número de publicações científicas e um quantitativo reduzido de autores que escrevem sobre a temática da resolução de situações problema foram enfrentados como limitações do estudo. A utilização dessa metodologia promove profissionais reflexivos e capazes de trabalhar em cooperação com os demais membros da equipe na construção de novos caminhos, possibilidades e práticas de modo a garantir a integralidade desses clientes. Sendo assim, surge a necessidade de novos estudos na área. 


\section{REFERÊNCIAS}

I. Universidade Federal do Paraná. Hospital de Clinicas. Diretoria de Enfermagem. Comissão de Sistematização da Assistência de Enfermagem (COMISAE). Avaliação de enfermagem: anamnese e exame físico (adulto, criança e gestante). Curitiba: Hospital de Clinica; 2014.

2. Patrício ACFA, Alves KL, Santos JS, Araruna PC, Duarte MCS, Rodrigues MMD. Exame físico cardiorrespiratório: conhecimento de estudantes de enfermagem. Rev Pesqui Cuid Fundam [Internet]. 2015 [acesso em: 20 jan 2018]; 7(1):1967-1974. Disponível em: http://www.seer.unirio.br/ index.php/cuidadofundamental/article/view/3525/pdf_I437

3. Carvalho V. Sobre o projeto para aplicação de novas metodologias ao processo ensino-aprendizagem: a experiência de mudança curricular na Graduação da EEAN/UFRJ. In: Carvalho V. Sobre enfermagem: ensino e perfil profissional. Rio de Janeiro (RJ): UFRJ/ EEAN; 2006. p. 159-75.

4. Carvalho V, Castro IB. Marco conceitual para o ensino e a pesquisa de enfermagem fundamental; um ponto de vista. Rev Bras Enferm [Internet]. 1985 [acesso em: 21 dez 2017]; 38(I):76-86. Disponível em: http://www.scielo.br/pdf/reben/v38n I/v38nla I I.pdf

5. Falco SM, Abdellah, FG. Teoria de enfermagem: dos fundamentos à prática profissional. $4^{\mathrm{a}}$ ed. Porto Alegre:Artes Médicas Sul; 2000.

6. Smeltzer SC, Bare BG. Hinkle JL, Cheever KH. Brunner \& Suddarth:Tratado de enfermagem médico-cirúrgica. 13 ed. Rio de Janeiro: Guanabara Koogan; 2015.

7. Raimundo DD, Guedes MTS, Luzial NS, Peixoto MGS, Santos MCM, Silva CC. Assistência de enfermagem a clientes com câncer na cabeça e no pescoço com ênfase nos tumores de cavidade oral no estado do Rio de Janeiro. Rev Pesqui Cuid Fundam [Internet]. 2014 [acesso em I4 mai 2018]; 6(4):I496-I504. Disponível em: http://www.seer. unirio.br/index.php/cuidadofundamental/article/viewFile/2088/pdf_979

8. Sousa RM, Espírito Santo FH, Pinheiro FM. Estudo de caso sobre as demandas de cuidados de enfermagem dos pacientes onco-hematológicos hospitalizados. Rev. enferm. UFPE [Internet]. 2017 [acesso em I4 mai 20l8]; II(I0):37963806. Disponível em: https://periodicos.ufpe.br/revistas/ revistaenfermagem/article/view/25190/24295

9. Barreto ABR, Haack A, Santos ACDS, Silva APRD. Perfil nutricional de pacientes pediátricos portadores de câncer, internados no Hospital da Criança de Brasília. Com. Ciências e Saúde [Internet] 2013 [acesso em 05 abr 20I8]; 24(4): 315 -
320. Disponível em : http://bvsms.saude.gov.br/bvs/artigos/ ccs/perfil_nutricional_pacientes_pediatricos_cancer.pdf

10. Ministério da Saúde (BR), Instituto Nacional de Câncer José Alencar Gomes da Silva, Coordenação de Prevenção e Vigilância. Estimativa 2018: incidência de câncer no Brasil [Internet]. Rio de Janeiro: Inca; 2017. Disponível em: http:// www.inca.gov.br/estimativa/20 I8/estimativa-2018.pdf

I I. Andrade V, Sawada NO, Barichello E. Qualidade de vida de pacientes com câncer hematológico em tratamento quimioterápico. Rev esc enferm USP [Internet] 2013 [acesso em I 2 jan 20 I8];47(2):355-36I. Disponível em: http://www. scielo.br/pdf/reeusp/v47n2/I2.pdf

12. Souza MAR;Wall ML; Moraes AC; et al. Poder vital e o legado de Florence Nightingale no processo saúde-doença: revisão integrativa. Rev Fund Care Online. [Internet] 2017 [acesso em 17 out 2018]; 9(I):297-30I. Disponível em : http://www.seer.unirio.br/index.php/cuidadofundamental/ article/view/4348/pdf_I

13. File W, Bylund CL, Kesselheim J, Leonard D, Leavey P. Do Pediatric Hematology/Oncology (PHO) fellows receive communication training? Pediatr Blood Cancer. [Internet] 2014 [ acesso em I5 out 20I8] ;6I(3):502-506. Disponivel em: https://www.ncbi.nlm.nih.gov/pubmed/24039096

14. Chibante CLP, Espírito Santo FH, Aquino ACO. The reactions of the family companion of hospitalized elderly facing stressful situations. Rev Pesqui Cuid Fundam [Internet]. 2015 [acesso em 20 jan 2018]; 7(3):296 I-73. Disponível em: http://www.seer.unirio.br/index.php/cuidadofundamental/ article/view/3899/pdf_1656

I5. Cecílio HPM, Arruda, GO, Marcon SS. A dependência do cuidado familiar na perspectiva do doente crônico. Rev Pesqui Cuid Fundam [Internet]. 2015 [acesso em 21 dez 2017]; 7(4): 1967-74]. Disponível em: http://www.seer.unirio.br/index.php/cuidadofundamental/article/view/3908/ pdf_1712

16. Costa VB, Camargo CR, Santos PHF, Lima LR, Stival MM, Funghetto SS. Avaliação da qualidade de vida e capacidade funcional de pacientes com câncer em tratamento quimioterápico. Cienc Cuid Saude [Internet]. 2017 [acesso em 02 mar 2018];16(3). Disponível em: http://www.periodicos. uem.br/ojs/index.php/CiencCuidSaude/article/view/35663

17. Galvão MIZ, Borges MS, Pinho DLM. Comunicação interpessoal com pacientes oncológicos em cuidados paliativos. Rev baiana enferm.[Internet]2017[ acesso em 18 out 20I8];3 I(3):e22290. Disponível em: https://portalseer.ufba. br/index.php/enfermagem/article/view/22290/I5092 